\title{
Spectral-Collocation Methods for Fractional Pantograph Delay-Integrodifferential Equations
}

\author{
Yin Yang ${ }^{1}$ and Yunqing Huang ${ }^{2}$ \\ ${ }^{1}$ College of Civil Engineering and Mechanics, Hunan Key Laboratory for Computation and Simulation in Science and Engineering, \\ Xiangtan University, Xiangtan, Hunan 411105, China \\ ${ }^{2}$ Hunan Key Laboratory for Computation and Simulation in Science and Engineering, Xiangtan University, \\ Xiangtan, Hunan 411105, China
}

Correspondence should be addressed to Yin Yang; yangyinxtu@xtu.edu.cn

Received 15 May 2013; Accepted 15 September 2013

Academic Editor: Varsha Daftardar-Gejji

Copyright (C) 2013 Y. Yang and Y. Huang. This is an open access article distributed under the Creative Commons Attribution License, which permits unrestricted use, distribution, and reproduction in any medium, provided the original work is properly cited.

We propose and analyze a spectral Jacobi-collocation approximation for fractional order integrodifferential equations of Volterra type with pantograph delay. The fractional derivative is described in the Caputo sense. We provide a rigorous error analysis for the collocation method, which shows that the error of approximate solution decays exponentially in $L^{\infty}$ norm and weighted $L^{2}$-norm. The numerical examples are given to illustrate the theoretical results.

\section{Introduction}

Many phenomena in engineering, physics, chemistry, and other sciences can be described very successfully by models using mathematical tools from fractional calculus, that is, the theory of derivatives and integrals of fractional noninteger order. This allows one to describe physical phenomena more accurately. Moreover, fractional calculus is applied to the model frequency dependent damping behavior of many viscoelastic materials, economics, and dynamics of interfaces between nanoparticles and substrates. Recently, several numerical methods to solve fractional differential equations (FDEs) and fractional integrodifferential equations (FIDEs) have been proposed.

In this paper, we consider the general linear fractional pantograph delay-integrodifferential equations (FDIDEs) with proportional delays,

$$
\begin{gathered}
D^{\gamma} y(t)=y(t)+y(q t)+\widehat{g}(t)+\int_{0}^{t} \widehat{K}_{1}(t, s) y(s) d s \\
+\int_{0}^{q t} \widehat{K}_{2}(t, \tau) y(\tau) d \tau, \\
0<\gamma \leq 1, t \in \widetilde{I}:=[0, T], \\
y(0)=y_{0},
\end{gathered}
$$

with $0<q<1$, where $\tilde{g}: \widetilde{I} \rightarrow R, \widehat{K}_{1}: D \rightarrow R(D:=$ $\{(t, \tau): 0 \leq s \leq t \leq T\})$, and $\widehat{K}_{2}: \widetilde{D} \rightarrow R(\widetilde{D}:=\{(t, \tau):$ $0 \leq \tau \leq q t, t \in \widetilde{I}\})$ are given functions and are assumed to be sufficiently smooth in the respective domains. In (1), $D^{\gamma}$ denotes the fractional derivative of fractional order $\gamma$.

Differential and integral equations involving derivatives of noninteger order have shown to be adequate models for various phenomena arising in damping laws, diffusion processes, models of earthquake [1], fluid-dynamics traffic model [2], mathematical physics and engineering [3], fluid and continuum mechanics [4], chemistry, acoustics, and psychology [5].

Let $\Gamma(\cdot)$ denote the Gamma function. For any positive integer $n$ and $n-1<\gamma<n$, the Caputo derivative is defined as follows:

$$
D^{\gamma} y(t)=\frac{1}{\Gamma(n-\gamma)} \int_{0}^{t} \frac{y^{(n)}(s)}{(t-s)^{(\gamma-n+1)}} d s, \quad t \in[0, T]
$$

The Riemann-Liouville fractional integral $I^{\gamma}$ of order $\gamma$ is defined as

$$
I^{\gamma} y(t)=\frac{1}{\Gamma(\gamma)} \int_{0}^{t}(t-s)^{\gamma-1} y(s) d s,
$$


we note that

$$
I^{\gamma}\left(D^{\gamma} y(t)\right)=y(t)-\sum_{k=0}^{n-1} y^{(k)}(0) \frac{t^{k}}{k !} .
$$

From (4), fractional integrodifferential equation (1) can be described as

$$
\begin{aligned}
D^{\gamma} y(t)= & y(t)+y(q t)+\widehat{g}(t)+\int_{0}^{t} \widehat{K}_{1}(t, s) y(s) d s \\
& +\int_{0}^{q t} \widehat{K}_{2}(t, \tau) y(\tau) d \tau, \\
0< & \gamma \leq 1, t \in \widetilde{I}:=[0, T], \\
y(t)= & \frac{1}{\Gamma(\gamma)} \int_{0}^{t}(t-\tau)^{\gamma-1} D^{\gamma} y(\tau) d \tau+y(0) .
\end{aligned}
$$

Several analytical methods have been introduced to solve FDEs including various transformation techniques [6], operational calculus methods [7], the Adomian decomposition method [8], and the iterative and series-based methods [9]. A small number of algorithms for the numerical solution of FDEs have been suggested [10], and most of them are finite difference methods, which are generally limited to low dimensions and are of limited accuracy.

As we know, fractional derivatives are global (they are defined by an integral over the whole interval $[0, T])$, and therefore, global methods such as spectral methods are perhaps better suited for FDEs. Standard spectral methods possess an infinite order of accuracy for the equations with regular solutions, while failing for many complicated problems with singular solutions. So, it is relevant to be interested in how to enlarge the adaptability of spectral methods and construct certain simple approximation schemes without a loss of accuracy for more complicated problems.

Spectral methods have been proposed to solve fractional differential equations, such as the Legendre collocation method [11, 12], Legendre wavelets method [13, 14], and Jacobi-Gauss-Lobatto collocation method [15]. The authors in [16-18] constructed an efficient spectral method for the numerical approximation of fractional integrodifferential equations based on tau and pseudospectral methods. Moreover, Bhrawy et al. [19] introduced a quadrature shifted Legendre tau method based on the Gauss-Lobatto interpolation for solving multiorder FDEs with variable coefficients and in [20], shifted Legendre spectral methods have been developed for solving fractional-order multipoint boundary value problems. In [21], truncated Legendre series together with the Legendre operational matrix of fractional derivatives are used for the numerical integration of fractional differential equations. In [22], the authors derived a new explicit formula for the integral of shifted Chebyshev polynomials of any degree for any fractional-order. The shifted Chebyshev operational matrix [23] and shifted Jacobi operational matrix [24] of fractional derivatives have been developed, which are applied together with the spectral tau method for numerical solution of general linear and nonlinear multiterm fractional differential equations. However, very few theoretical results were provided to justify the high accuracy numerically obtained. Recently, Chen and Tang $[25,26]$ developed a novel spectral Jacobi-collocation method to solve second kind Volterra integral equations with a weakly singular kernel and provided a rigorous error analysis which theoretically justifies the spectral rate of convergence. Inspired by the work of [26], we extend the approach to fractional order delay-integrodifferential equations (1). However, it is difficult to apply the spectral approximations to the initial value problem and fractional order derivatives. To facilitate the use of the spectral methods, we restate the initial condition as an equivalent integral equation with singular kernel. Then, we get the discrete scheme by using Gauss quadrature formula. In this paper, we will provide a rigorous error analysis not only for approximate solutions but also for approximate fractional derivatives which theoretically justifies the spectral rate of convergence.

For ease of analysis, we will describe the spectral methods on the standard interval $I:=[-1,1]$. Hence, we employ the transformation

$$
t=\frac{T}{2}(1+x), \quad s=\frac{T(1+\theta)}{2}, \quad \tau=\frac{T(1+\eta)}{2}
$$

then, the previous problem (5) becomes

$$
\begin{aligned}
& D^{\gamma} u(x)= u(x)+u(q x+q-1)+g(x) \\
&+\int_{-1}^{x} K_{1}(x, \theta) u(\theta) d \theta \\
&+\int_{-1}^{q x+q-1} K_{2}(x, \eta) u(\eta) d \eta, \quad x \in I, \\
& u(x)=\frac{1}{\Gamma(\gamma)}\left(\frac{T}{2}\right)^{\gamma} \int_{-1}^{x}(x-s)^{\gamma-1} D^{\gamma} u(s) d s+u(-1),
\end{aligned}
$$

where

$$
\begin{gathered}
u(x)=y\left(\frac{T}{2}(1+x)\right), \quad D^{\gamma} u(x)=D^{\gamma} y\left(\frac{T}{2}(1+x)\right) \\
g(x)=\frac{T}{2} \widehat{g}\left(\frac{T}{2}(1+x)\right) \\
K_{1}(x, \theta)=\frac{T}{4} \widehat{K}_{1}\left(\frac{T}{2}(1+x), \frac{T}{2}(1+\theta)\right) \\
K_{2}(x, \eta)=\frac{T}{4} \widehat{K}_{2}\left(\frac{T}{2}(1+x), \frac{T}{2}(1+\eta)\right)
\end{gathered}
$$

This paper is organized as follows. In Section 2, we introduce the spectral approaches for pantograph FDIDEs. Some useful lemmas are provided in Section 3. These lemmas will play a key role in the derivation of the convergence analysis. We provide a rigorous error analysis for the spectral methods, which shows that both the errors of approximate solutions and the errors of approximate fractional derivatives of the solutions decay exponentially in $L^{\infty}$ norm and weighted $L^{2}$ norm in Section 4, and Section 5 contains numerical results, 
which will be used to verify the theoretical results obtained in Section 4 .

Throughout the paper, $C$ will denote a generic positive constant that is independent of $N$ but which will depend on $q, T$ and on the bounds for the given functions $a, b$, and $K_{j}$, $j=1,2$.

\section{Jacobi-Collocation Method}

Let $\omega^{\alpha, \beta}(x)=(1-x)^{\alpha}(1+x)^{\beta}$ be a weight function in the usual sense, for $\alpha, \beta>-1$. The set of Jacobi polynomials $\left\{J_{n}^{\alpha, \beta}(x)\right\}_{n=0}^{\infty}$ forms a complete $L_{\omega^{\alpha, \beta}}^{2}(-1,1)$-orthogonal system, where $L_{\omega^{\alpha, \beta}}^{2}(-1,1)$ is a weighted space defined by

$$
L_{\omega^{\alpha, \beta}}^{2}(-1,1)=\left\{v: v \text { is measurable and }\|v\|_{\omega^{\alpha, \beta}}<\infty\right\} \text {, }
$$

equipped with the norm

$$
\|v\|_{\omega^{\alpha, \beta}}=\left(\int_{-1}^{1}|v(x)|^{2} \omega^{\alpha, \beta}(x) d x\right)^{1 / 2}
$$

and the inner product

$$
(u, v)_{\omega^{\alpha, \beta}}=\int_{-1}^{1} u(x) v(x) \omega^{\alpha, \beta}(x) d x, \quad \forall u, v \in L_{\omega^{\alpha, \beta}}^{2}(-1,1) .
$$

For a given $N \geq 0$, we denote by $\left\{\tilde{\theta}_{k}\right\}_{k=0}^{N}$ the Legendre points and by $\left\{\omega_{k}\right\}_{k=0}^{N}$ the corresponding Legendre weights (i.e., Jacobi weights $\left\{\omega_{k}^{0,0}\right\}_{k=0}^{N}$ ). Then, the Legendre-Gauss integration formula is

$$
\int_{-1}^{1} f(x) d x \approx \sum_{k=0}^{N} f\left(\theta_{k}\right) \omega_{k}
$$

Similarly, we denote by $\left\{\theta_{k}\right\}_{k=0}^{N}$ the Jacobi-Gauss points and by $\left\{\omega_{k}^{\alpha, \beta}\right\}_{k=0}^{N}$ the corresponding Jacobi weights. Then, the JacobiGauss integration formula is

$$
\int_{-1}^{1} f(x) \omega^{\alpha, \beta}(x) d x \approx \sum_{k=0}^{N} f\left(\widetilde{\theta}_{k}\right) \omega_{k}^{\alpha, \beta}
$$

For a given positive integer $N$, we denote the collocation points by $\left\{x_{i}^{\alpha, \beta}\right\}_{i=0}^{N}$, which is the set of $(N+1)$ Jacobi-Gauss points corresponding to the weight $\omega^{\alpha, \beta}(x)$. Let $\mathscr{P}_{N}$ denote the space of all polynomials of degree not exceeding $N$. For any $v \in C[-1,1]$, we can define the Lagrange interpolating polynomial $I_{N}^{\alpha, \beta} v \in \mathscr{P}_{N}$, satisfying

$$
I_{N}^{\alpha, \beta} v\left(x_{i}\right)=v\left(x_{i}\right), \quad 0 \leq i \leq N .
$$

The Lagrange interpolating polynomial can be written in the form

$$
I_{N}^{\alpha, \beta} v(x)=\sum_{i=0}^{N} v\left(x_{i}\right) F_{i}(x), \quad 0 \leq i \leq N
$$

where $F_{i}(x)$ is the Lagrange interpolation basis function associated with $\left\{x_{i}\right\}_{i=0}^{N}$.

Let $-\mu=\gamma-1$. In order to obtain high order accuracy of the approximate solution, the main difficulty is to compute the integral terms in (7) and (8). In particular, for small values of $x$, there is little information available for $u$. To overcome this difficulty, we transfer the integration interval to a fixed interval $[-1,1]$ by using the following variable changes

$$
\begin{gathered}
\theta=\frac{1+x}{2} \nu+\frac{x-1}{2} \triangleq \theta(x, \nu), \\
\eta=\frac{q(1+x)}{2} \xi+\frac{q(1+x)}{2}-1 \triangleq \eta(x, \xi), \\
D^{\gamma} u(x)=u(x)+u(q x+q-1)+g(x) \\
+\frac{1+x}{2} \int_{-1}^{1} K_{1}(x, \theta(x, \nu)) u(\theta(x, \nu)) d \nu \\
+\frac{q(1+x)}{2} \int_{-1}^{1} K_{2}(x, \eta(x, \xi)) u(\eta(x, \xi)) d \xi,
\end{gathered}
$$

$$
\begin{aligned}
u(x)= & \frac{1}{\Gamma(\gamma)}\left(\frac{1+x}{2}\right)^{\gamma} \int_{-1}^{1}(1-\nu)^{-\mu} D^{\gamma} u \\
& \times(\theta(x, \nu)) d \nu+u(-1)
\end{aligned}
$$

Set the collocation points as the set of $(N+1)$ JacobiGauss points, $\left\{x_{i}^{-\mu,-\mu}\right\}_{i=0}^{N}$ associated with $\omega^{-\mu,-\mu}$. Assume that (19) and (20) holds at $x_{i}^{-\mu,-\mu}$ :

$$
\begin{aligned}
& D^{\gamma} u\left(x_{i}^{-\mu,-\mu}\right)=u\left(x_{i}^{-\mu,-\mu}\right)+u\left(q x_{i}^{-\mu,-\mu}+q-1\right) \\
& +g\left(x_{i}^{-\mu,-\mu}\right)+\frac{1+x_{i}^{-\mu,-\mu}}{2} \\
& \times \int_{-1}^{1} K_{1}\left(x_{i}^{-\mu,-\mu}, \theta\left(x_{i}^{-\mu,-\mu}, \nu\right)\right) \\
& \times u\left(\theta\left(x_{i}^{-\mu,-\mu}, v\right)\right) d v \\
& +\frac{q\left(1+x_{i}^{-\mu,-\mu}\right)}{2} \\
& \times \int_{-1}^{1} K_{2}\left(x_{i}^{-\mu,-\mu}, \eta\left(x_{i}^{-\mu,-\mu}, \xi\right)\right) \\
& \times u\left(\eta\left(x_{i}^{-\mu,-\mu}, \xi\right)\right) d \xi, \\
& u\left(x_{i}^{-\mu,-\mu}\right)=\frac{1}{\Gamma(\gamma)}\left(\frac{\left(1+x_{i}^{-\mu,-\mu}\right) T}{4}\right)^{\gamma} \\
& \times \int_{-1}^{1}(1-v)^{-\mu} D^{\gamma} u\left(\theta\left(x_{i}^{-\mu,-\mu}, v\right)\right) d \nu \\
& +u(-1) \text {. }
\end{aligned}
$$


Next, using a $(N+1)$-point Gauss quadrature formula relative to the Jacobi weight $\left\{\omega_{k}^{0,0}\right\}_{i=0}^{N}$, the integration term in (21) can be approximated by

$$
\begin{gathered}
\int_{-1}^{1} K_{1}\left(x^{-\mu,-\mu}, \theta\left(x_{i}^{-\mu,-\mu}, \nu\right)\right) u\left(\theta\left(x_{i}^{-\mu,-\mu}, \nu\right)\right) d \nu \\
=\sum_{k=0}^{N} K_{1}\left(x_{i}, \theta\left(x_{i}, v_{k}\right)\right) u\left(\theta\left(x_{i}, v_{k}\right)\right) \omega_{k}^{0,0}, \\
\int_{-1}^{1} K_{2}\left(x_{i}^{-\mu,-\mu}, \eta\left(x_{i}^{-\mu,-\mu}, \xi\right)\right) u\left(\eta\left(x_{i}^{-\mu,-\mu}, \xi\right)\right) d \xi \\
=\sum_{k=0}^{N} K_{2}\left(x_{i}, \eta\left(x_{i}, \xi_{k}\right)\right) u\left(\eta\left(x_{i}, \xi_{k}\right)\right) \omega_{k}^{0,0} .
\end{gathered}
$$

The sets $\left\{v_{k}\right\}_{k=0}^{N}$ and $\left\{\xi_{k}\right\}_{k=0}^{N}$ coincide with the Jacobi-Gauss points corresponding Jacobi weights $\left\{\omega_{k}^{0,0}\right\}_{k=0}^{N}$; that is, $\left\{v_{k}\right\}_{k=0}^{N}$ and $\left\{\xi_{k}\right\}_{k=0}^{N}$ are Legendre-Gauss points.

Using a $(N+1)$-point Gauss quadrature formula relative to the Jacobi weight $\omega^{-\mu, 0}$, the integration term in (22) can be approximated by

$$
\begin{aligned}
\int_{-1}^{1}(1-v)^{\mu} D^{\gamma} u\left(\theta\left(x_{i}^{-\mu,-\mu}, \nu\right)\right) d \theta \\
=\sum_{k=0}^{N} D^{\gamma} u\left(\theta\left(x_{i}^{-\mu,-\mu}, \widetilde{v}_{k}\right)\right) \omega_{k}^{-\mu, 0},
\end{aligned}
$$

where the set $\left\{\widetilde{v}_{k}\right\}_{k=0}^{N}$ is the Jacobi-Gauss points corresponding to the weight $\omega^{-\mu, 0}$.

We use $u_{i}, u_{i}^{\gamma}$ to approximate the function value $u\left(x_{i}^{-\mu,-\mu}\right)$, $D^{\gamma} u\left(x_{i}^{-\mu,-\mu}\right), 0 \leq i \leq N$, and expand $u$ and $u^{\gamma}$ using Lagrange interpolation polynomials; that is,

$$
U(x)=\sum_{j=0}^{N} u_{j} F_{j}(x), \quad U^{\gamma}(x)=\sum_{j=0}^{N} u_{j}^{\gamma} F_{j}(x),
$$

where $F_{j}(x)$ is the Lagrange interpolation basis function associated with $\left\{x_{i}^{-\mu,-\mu}\right\}_{i=0}^{N}$ which is the set of $(N+1)$ Jacobi-Gauss points. The Jacobi collocation methods are to seek $\left\{u_{i}^{\gamma}\right\}_{i=0}^{N}$ and $\left\{u_{i}\right\}_{i=0}^{N}$ such that the following collocation equations hold:

$$
\begin{aligned}
u_{i}^{\gamma}= & u_{i}+\left(\sum_{j=0}^{N} u_{j} F_{j}\left(q x_{i}^{-\mu,-\mu}+q-1\right)\right) \\
+ & g\left(x_{i}^{-\mu,-\mu}\right) \\
+ & \frac{1+x_{i}^{-\mu,-\mu}}{2} \sum_{j=0}^{N} u_{j}\left(\sum_{k=0}^{N} K_{1}\left(x_{i}^{-\mu,-\mu}, \theta\left(x_{i}, v_{k}\right)\right)\right. \\
& \left.\times F_{j}\left(\theta\left(x_{i}^{-\mu,-\mu}, v_{k}\right)\right) \omega_{k}^{0,0}\right)
\end{aligned}
$$

$$
+\frac{q\left(1+x_{i}^{-\mu,-\mu}\right)}{2} \sum_{j=0}^{N} u_{j}\left(\sum_{k=0}^{N} K_{2}\left(x_{i}^{-\mu,-\mu}, \eta\left(x_{i}, v_{k}\right)\right)\right.
$$$$
\left.\times F_{j}\left(\eta\left(x_{i}^{-\mu,-\mu}, v_{k}\right)\right) \omega_{k}^{0,0}\right),
$$

$$
\begin{aligned}
u_{i}= & \frac{1}{\Gamma(\gamma)}\left(\frac{\left(1+x_{i}^{-\mu,-\mu}\right) T}{4}\right)^{\gamma} \sum_{j=0}^{N} u_{j}^{\gamma} \\
& \times\left(\sum_{k=0}^{N} F_{j}\left(s\left(x_{i}^{-\mu,-\mu}, \widetilde{v}_{k}\right)\right) \omega_{k}^{-\mu, 0}\right)+u(-1) .
\end{aligned}
$$

Writing $U_{N}=\left(u_{0}, u_{1}, \ldots, u_{N}\right)^{T}$ and $U_{N}^{\gamma}=\left(u_{0}^{\gamma}, u_{1}^{\gamma}, \ldots\right.$, $\left.u_{N}^{\gamma}\right)^{T}$, we obtain the following of the matrix form from (26)(27):

$$
\begin{gathered}
U_{N}^{\gamma}=(E+L+C+D) U_{N}+G, \\
U_{N}=U_{-1}+B U_{N}^{\gamma},
\end{gathered}
$$

where

$E$ is the identity matrix,

$$
\begin{aligned}
& G=\left(\widetilde{g}\left(x_{0}^{-\mu,-\mu}\right), \widetilde{g}\left(x_{1}^{-\mu,-\mu}\right), \ldots, \widetilde{g}\left(x_{N}^{-\mu,-\mu}\right)\right)^{T}, \\
& L_{i j}=F_{j}\left(q x_{i}^{-\mu,-\mu}+q-1\right) \text {, } \\
& C_{i j}=\frac{1+x_{i}^{-\mu,-\mu}}{2} \sum_{k=0}^{N} \widetilde{K}_{1}\left(x_{i}^{-\mu,-\mu}, \theta\left(x_{i}^{-\mu,-\mu}, v_{k}\right)\right) F_{j} \\
& \times\left(\theta\left(x_{i}, v_{k}\right)\right) \omega_{k}^{0,0}, \\
& D_{i j}=\frac{q\left(1+x_{i}^{-\mu,-\mu}\right)}{2} \sum_{k=0}^{N} \widetilde{K}_{2}\left(x_{i}^{-\mu,-\mu}, \eta\left(x_{i}^{-\mu,-\mu}, v_{k}\right)\right) F_{j} \\
& \times\left(\eta\left(x_{i}^{-\mu,-\mu}, v_{k}\right)\right) \omega_{k}^{0,0}, \\
& B_{i j}=\frac{1}{\Gamma(\gamma)}\left(\frac{\left(1+x_{i}^{-\mu,-\mu}\right) T}{4}\right)^{\gamma} \\
& \times \sum_{k=0}^{N} F_{j}\left(s\left(x_{i}^{-\mu,-\mu}, \widetilde{v}_{k}\right)\right) \omega_{k}^{-\mu, 0}, \\
& U_{-1}=(u(-1), u(-1), \ldots, u(-1))^{T} \text {. }
\end{aligned}
$$

We can get the values of $\left\{u_{i}\right\}_{i=0}^{N}$ and $\left\{u_{i}^{\gamma}\right\}_{i=0}^{N}$ by solving the system of linear system (28). Therefore, the expressions of $U(x)$ and $U^{\gamma}(x)$ can be obtained.

\section{Some Useful Lemmas}

In this section, we will provide some elementary lemmas, which are important for the derivation of the main results in the subsequent section. Let $I:=(-1,1)$. 
Lemma 1 (see [27]). Assume that an $(N+1)$-point Gauss quadrature formula relative to the Jacobi weight is used to integrate the product $u \varphi$, where $u \in H^{m}(I)$ with I for some $m \geq$ 1 and $\varphi \in \mathscr{P}_{N}$. Then, there exists a constant $C$ independent of $N$ such that

$$
\left|\int_{-1}^{1} u(x) \varphi(x) d x-(u, \varphi)_{N}\right| \leq C N^{-m}|u|_{H^{\alpha, \beta}}^{m, N}(I)\|\varphi\|_{L_{\omega^{\alpha, \beta}}^{2}(I)},
$$

where

$$
\begin{gathered}
|u|_{H^{\prime, \beta}, N}^{m, N}(I)=\left(\sum_{j=\min (m, N+1)}^{m}\left\|u^{(j)}\right\|_{L_{\omega^{\alpha, \beta}}^{2}(I)}^{2}\right)^{1 / 2}, \\
(u, \varphi)_{N}=\sum_{j=0}^{N} u\left(x_{j}\right) \varphi\left(x_{j}\right) \omega_{j} .
\end{gathered}
$$

Lemma 2 (see [26, 27]). Assume that $u \in H_{\omega^{-\mu,-\mu}}^{m, N}(I)$ and denote by $I_{N}^{-\mu,-\mu} u$ its interpolation polynomial associated with the $(N+1)$ Jacobi-Gauss points $\left\{x_{j}\right\}_{j=0}^{N}$; namely,

$$
I_{N}^{-\mu,-\mu} u=\sum_{i=0}^{N} u\left(x_{i}\right) F\left(x_{i}\right)
$$

Then, the following estimates hold:

$$
\begin{aligned}
& \left\|u-I_{N}^{-\mu,-\mu} u\right\|_{L_{\omega^{-\mu,-\mu}}^{2}(I)} \leq C N^{-m}|u|_{H_{\omega^{\alpha, \beta}}^{m, N}(I)}, \\
\left\|u-I_{N}^{-\mu,-\mu} u\right\|_{L^{\infty}(I)} & \begin{cases}C N^{1-\mu-m}|u|_{H_{\omega^{c}}^{m, N}(I)}, & 0 \leq \mu<\frac{1}{2}, \\
C N^{1 / 2-m} \log N|u|_{H_{\omega^{c}}^{m, N}(I)}, & \frac{1}{2} \leq \mu<1,\end{cases}
\end{aligned}
$$

where $\omega^{c}=\omega^{-1 / 2,-1 / 2}$ denotes the Chebyshev weight function.

Lemma 3 (see [28]). Assume that $\left\{F_{j}(x)\right\}_{j=0}^{N}$ are the $N$-th degree Lagrange basis polynomials associated with the Gauss points of the Jacobi polynomials. Then,

$$
\begin{aligned}
\left\|I_{N}^{\alpha, \beta}\right\|_{L^{\infty}(I)} & \leq \max _{x \in[-1,1]} \sum_{j=0}^{N}\left|F_{j}(x)\right| \\
& = \begin{cases}\mathcal{O}(\log N), & -1<\alpha, \beta \leq-\frac{1}{2}, \\
\mathcal{O}\left(N^{\gamma+1 / 2}\right), & \gamma=\max (\alpha, \beta), \text { otherwise. }\end{cases}
\end{aligned}
$$

Lemma 4 (Gronwall inequality, see [29] Lemma 7.1.1). Suppose that $L \geq 0,0<\mu<1$, and $u$ and $v$ are a nonnegative, locally integrable functions defined on $[-1,1]$ satisfying

$$
u(x) \leq v(x)+L \int_{-1}^{x}(x-\tau)^{-\mu} u(\tau) d \tau
$$

Then, there exists a constant $C=C(\mu)$ such that

$$
u(x) \leq v(x)+C L \int_{-1}^{x}(x-\tau)^{-\mu} v(\tau) d \tau, \quad \text { for }-1 \leq x<1
$$

Lemma 5 (see $[30,31])$. For a nonnegative integer $r$ and $\kappa \epsilon$ $(0,1)$, there exists a constant $C_{r, \kappa}>0$ such that for any function $v \in C^{r, \kappa}([-1,1])$, there exists a polynomial function $\mathscr{T}_{N} v \in$ $\mathscr{P}_{N}$ such that

$$
\left\|v-\mathscr{T}_{N} v\right\|_{L^{\infty}(I)} \leq C_{r, \kappa} N^{-(r+\kappa)}\|v\|_{r, \kappa},
$$

where $\|\cdot\|_{r, \kappa}$ is the standard norm in $C^{r, \kappa}([-1,1])$ which is denoted by the space of functions whose rth derivatives are Hölder continuous with exponent $\kappa$, endowed with the usual norm

$$
\begin{aligned}
\|v\|_{r, \kappa}= & \max _{0 \leq \kappa \leq r} \max _{x \in[-1,1]}\left|\partial_{x}^{\kappa} v(x)\right| \\
& +\max _{0 \leq \kappa \leq r} \sup _{x, y \in[-1,1], x \neq y} \frac{\left|\partial_{x}^{\kappa} v(x)-\partial_{x}^{\kappa} v(x)\right|}{|x-y|^{\kappa}} .
\end{aligned}
$$

$\mathscr{T}_{N}$ is a linear operator from $C^{r, \kappa}([-1,1])$ into $\mathscr{P}_{N}$.

Lemma 6 (see [32]). Let $\kappa \in(0,1)$ and let $\mathscr{M}$ be defined by

$$
(\mathscr{M} v)(x)=\int_{-1}^{x}(x-\tau)^{-\mu} K(x, \tau) v(\tau) d \tau
$$

Then, for any function $v \in C([-1,1])$, there exists a positive constant $C$ such that

$$
\frac{\left|\mathscr{M} v\left(x^{\prime}\right)-\mathscr{M} v\left(x^{\prime \prime}\right)\right|}{\left|x^{\prime}-x^{\prime \prime}\right|} \leq C \max _{x \in[-1,1]}|v(x)|,
$$

under the assumption that $0<\kappa<1-\mu$, for any $x^{\prime}, x^{\prime \prime} \in$ $[-1,1]$ and $x^{\prime} \neq x^{\prime \prime}$. This implies that

$$
\|\mathscr{M} v\|_{0, \kappa} \leq C \max _{x \in[-1,1]}|v(x)|, \quad 0<\kappa<1-\mu .
$$

Lemma 7 (see [33]). For every bounded function $v$, there exists a constant $C$, independent of $v$ such that

$$
\sup _{N}\left\|\sum_{j=0}^{N} v\left(x_{j}\right) F_{j}(x)\right\|_{L_{\omega^{\alpha}, \beta}^{2}(I)} \leq C \max _{x \in[-1,1]}|v(x)|,
$$

where $F_{j}(x), j=0,1, \ldots, N$, are the Lagrange interpolation basis functions associated with the Jacobi collocation points $\left\{x_{j}\right\}_{j=0}^{N}$.

Lemma 8 (see [34]). For all measurable function $f \geq 0$, the following generalized Hardy's inequality

$$
\left(\int_{a}^{b}|(T f)(x)|^{q} u(x) d x\right)^{1 / q} \leq\left(\int_{a}^{b}|f(x)|^{p} v(x) d x\right)^{1 / p}
$$


holds if and only if

$$
\begin{gathered}
\sup _{a<x<b}\left(\int_{x}^{b} u(t) d t\right)^{1 / q}\left(\int_{a}^{x} v^{1-p^{\prime}}(t) d t\right)^{1 / p^{\prime}} \\
\quad<\infty, \quad p^{\prime}=\frac{p}{p-1}
\end{gathered}
$$

for the case $1<p \leq q<\infty$. Here, $T$ is an operator of the form

$$
(T F)(x)=\int_{a}^{x} k(x, t) f(t) d t,
$$

with $k(x, t)$ a given kernel, $u, v$ are nonnegative weight functions, and $-\infty \leq a<b \leq \infty$.

\section{Convergence Analysis}

This section is devoted to provide a convergence analysis for the numerical scheme. The goal is to show that the rate of convergence is exponential; that is, the spectral accuracy can be obtained for the proposed approximations. Firstly, we will carry our convergence analysis in $L^{\infty}$ space.

Theorem 9. Let $u(x)$ be the exact solution of the fractional delay-integrodifferential equation (7)-(8), which is assumed to be sufficiently smooth. Assume that $U(x)$ and $U^{\gamma}(x)$ are obtained by using the spectral collocation scheme (26)-(27) together with a polynomial interpolation (25). If $\gamma$ associated with the weakly singular kernel satisfies $0<\gamma<1$ and $u \in H_{\omega^{-\mu,-\mu}}^{m+1}(I)$, then

$$
\begin{aligned}
\| U^{\gamma} & -D^{\gamma} u \|_{L^{\infty}(I)} \\
& \leq \begin{cases}C N^{\gamma-1 / 2-m}\left(K^{*}\|u\|_{L^{2}(I)}+N^{1 / 2} \mathcal{U}\right), & \frac{1}{2}<\gamma<1, \\
C N^{-m} \log N\left(K^{*}\|u\|_{L^{2}(I)}+N^{1 / 2} \mathcal{U}\right), & 0<\gamma \leq \frac{1}{2},\end{cases} \\
\| U & -u \|_{L^{\infty}(I)} \\
& \leq \begin{cases}C N^{\gamma-1 / 2-m}\left(K^{*}\|u\|_{L^{2}(I)}+N^{1 / 2} U\right), & \frac{1}{2}<\gamma<1, \\
C N^{-m} \log N\left(K^{*}\|u\|_{L^{2}(I)}+N^{1 / 2} U\right), & 0<\gamma \leq \frac{1}{2},\end{cases}
\end{aligned}
$$

provided that $N$ is sufficiently large, where $C$ is a constant independent of $N$ but which will depend on the bounds of the functions $K(x, s)$ and the index $\mu$,

$$
\begin{aligned}
K^{*}= & \max _{x \in[-1,1]}\left|K_{1}(x, \theta(x, \cdot))\right|_{\widetilde{H}_{\omega^{0}, 0}^{m, N}(I)} \\
& +\max _{x \in[-1,1]}\left|K_{2}(x, \eta(x, \cdot))\right|_{\widetilde{H}_{\omega, 0}^{m, N}(I)}, \\
\mathscr{U} & =\left|D^{\gamma} u\right|_{H_{\omega^{c}}^{m, N}(I)}+|u|_{H_{\omega^{0}}^{m, N}(I)} .
\end{aligned}
$$

Proof. We let

$$
\begin{aligned}
& \left(K\left(s\left(x_{i}, v\right)\right), \phi\left(s\left(x_{i}, v\right)\right)\right)_{N} \\
& \quad=\sum_{k=0}^{N} K\left(s\left(x_{i}, v_{k}\right)\right) \phi\left(s\left(x_{i}, v_{k}\right)\right) \omega_{k}^{0,0} .
\end{aligned}
$$

The numerical scheme (26)-(27) can be written as

$$
\begin{aligned}
u_{i}^{\gamma}= & u_{i}+\left(\sum_{j=0}^{N} u_{j} F_{j}\left(q x_{i}^{-\mu,-\mu}+q-1\right)\right)+g\left(x_{i}^{-\mu,-\mu}\right) \\
& +\frac{1+x_{i}^{-\mu,-\mu}}{2}\left(K_{1}\left(x_{i}^{-\mu,-\mu}, \theta\left(x_{i}^{-\mu,-\mu}, \nu\right)\right),\right. \\
& \left.U\left(\theta\left(x_{i}^{-\mu,-\mu}, \nu\right)\right)\right)_{N} \\
& +\frac{q\left(1+x_{i}^{-\mu,-\mu}\right)}{2}\left(K_{2}\left(x_{i}^{-\mu,-\mu}, \eta\left(x_{i}^{-\mu,-\mu}, \nu\right)\right),\right. \\
u_{i}= & \left.\left.\left.\frac{1}{\Gamma(\gamma)}\left(\frac{T}{2}\right)^{\gamma} \int_{-1}^{x_{i}}\left(x_{i}-\theta\right)^{-\mu} U^{-\mu,-\mu}, \nu\right)\right)\right)_{N},
\end{aligned}
$$

which gives

$$
\begin{aligned}
u_{i}^{\gamma}= & u_{i}+\left(\sum_{j=0}^{N} u_{j} F_{j}\left(q x_{i}^{-\mu,-\mu}+q-1\right)\right)+g\left(x_{i}^{-\mu,-\mu}\right) \\
& +\frac{1+x_{i}^{-\mu,-\mu}}{2} \int_{-1}^{1} K_{1}\left(x_{i}^{-\mu,-\mu}, \theta\left(x_{i}^{-\mu,-\mu}, \nu\right)\right) \\
& \times U\left(\theta\left(x_{i}^{-\mu,-\mu}, \nu\right)\right) d \nu+I_{1}\left(x_{i}^{-\mu,-\mu}\right) \\
& +\frac{q\left(1+x_{i}^{-\mu,-\mu}\right)}{2} \int_{-1}^{1} K_{2}\left(x_{i}^{-\mu,-\mu}, \eta\left(x_{i}^{-\mu,-\mu}, \xi\right)\right) \\
& \times U\left(\eta\left(x_{i}^{-\mu,-\mu}, \xi\right)\right) d \xi+I_{2}\left(x_{i}^{-\mu,-\mu}\right),
\end{aligned}
$$

where

$$
\begin{aligned}
I_{1}(x)= & \frac{1+x}{2} \int_{-1}^{1} K_{1}(x, \theta(x)), U(\theta(x, \nu)) d \nu \\
& -\frac{1+x}{2}\left(K_{1}(x, \theta(x, \nu)), U(\theta(x, \nu))\right)_{N}, \\
I_{2}(x)= & \frac{q(1+x)}{2} \int_{-1}^{1} K_{2}(x, \eta(x, \xi)) U(\eta(x, \xi)) d \xi \\
& -\frac{q(1+x)}{2}\left(K_{2}(x, \eta(x, \nu)), U(\eta(x, \nu))\right)_{N} .
\end{aligned}
$$


Using the integration error estimates from Jacobi-Gauss polynomials quadrature in Lemma 1, we have

$$
\begin{aligned}
&\left|I_{1}(x)\right| \leq C N^{-m}\left|\widetilde{K}_{1}(x, \theta(x, \cdot))\right|_{H_{\omega^{0,0}}^{m ; N}(I)}\|U(\theta(x, \cdot))\|_{L^{2}(I)} \\
& \leq C N^{-m} K_{1}^{*}\|U\|_{L^{2}(I)}, \\
&\left|I_{2}(x)\right| \leq C N^{-m}\left|\widetilde{K}_{2}(x, \eta(x, \cdot))\right|_{H_{\omega^{0,0}}^{m ; N}(I)}\|U(\eta(x, \cdot))\|_{L^{2}(I)} \\
& \leq C N^{-m} K_{2}^{*}\|U\|_{L^{2}(I)}, \\
& K_{1}^{*}=\max _{x \in[-1,1]}\left|K_{1}(x, \theta(x, \cdot))\right|_{\widetilde{H}_{\omega^{0,0}}^{m, N}(I)}, \\
& K_{2}^{*}=\max _{x \in[-1,1]}\left|K_{1}(x, \theta(x, \cdot))\right|_{\widetilde{H}_{\omega^{0,0}}^{m, N}(I)} .
\end{aligned}
$$

From (18), (53) can be rewritten as

$$
\begin{aligned}
u_{i}^{\gamma}= & a\left(x_{i}^{-\mu,-\mu}\right) u_{i}+b\left(x_{i}^{-\mu,-\mu}\right) \\
& \times\left(\sum_{j=0}^{N} u_{j} F_{j}\left(q x_{i}^{-\mu,-\mu}+q-1\right)\right)+g\left(x_{i}^{-\mu,-\mu}\right) \\
& +\int_{-1}^{x_{i}^{-\mu,-\mu}} K_{1}\left(x_{i}^{-\mu,-\mu}, \theta\right) U(\theta) d \theta+I_{1}\left(x_{i}^{-\mu,-\mu}\right) \\
& +\int_{-1}^{q x_{i}^{-\mu,-\mu}+q-1} K_{2}\left(x_{i}^{-\mu,-\mu}, \eta\right) U(\eta) d \eta \\
& +I_{2}\left(x_{i}^{-\mu,-\mu}\right) .
\end{aligned}
$$

Let $e$ and $e^{\gamma}$ denote the error function

$$
e(x)=U(x)-u(x), \quad e^{\gamma}(x)=U^{\gamma}(x)-u^{\gamma}(x),
$$

we have

$$
\begin{aligned}
u_{i}^{\gamma}= & a\left(x_{i}^{-\mu,-\mu}\right) u_{i}+b\left(x_{i}^{-\mu,-\mu}\right) \\
& \times\left(\sum_{j=0}^{N} u_{j} F_{j}\left(q x_{i}^{-\mu,-\mu}+q-1\right)\right)+g\left(x_{i}^{-\mu,-\mu}\right) \\
& +\int_{-1}^{x_{i}^{-\mu,-\mu}} K_{1}\left(x_{i}^{-\mu,-\mu}, \theta\right) u(\theta) d \theta \\
& +\int_{-1}^{x_{i}^{-\mu,-\mu}} K_{1}\left(x_{i}^{-\mu,-\mu}, \theta\right) e(\theta) d \theta \\
& +\int_{-1}^{q x_{i}^{-\mu,-\mu}+q-1} K_{2}\left(x_{i}^{-\mu,-\mu}, \eta\right) u(\eta) d \eta \\
& +\int_{-1}^{q x_{i}^{-\mu,-\mu}+q-1} K_{2}\left(x_{i}^{-\mu,-\mu}, \eta\right) e(\eta) d \eta \\
& +I_{1}\left(x_{i}^{-\mu,-\mu}\right)+I_{2}\left(x_{i}^{-\mu,-\mu}\right) .
\end{aligned}
$$

Multiplying $F_{i}(x)$ on both sides of (62) and (52), summing up from 0 to $N$, and using (7)-(8) yield

$$
\begin{aligned}
U^{\gamma}(x)= & U(x)+I_{N}^{-\mu,-\mu}\left(\sum_{j=0}^{N} u_{j} F_{j}(q x+q-1)\right) \\
& +I_{N}^{-\mu,-\mu} g(x) \\
& +I_{N}^{-\mu,-\mu}\left[D^{\gamma} u(x)-u(x)\right. \\
& +I_{N}^{-\mu,-\mu} \int_{-1}^{x} K_{1}(x, \theta) e(\theta) d \theta \\
& +I_{N}^{-\mu,-\mu} \int_{-1}^{q x+q-1} K_{2}(x, \eta) e(\eta) d \eta \\
& +J_{1}(x)+J_{2}(x), \\
= & I_{N}^{-\mu,-\mu}\left(u(x)-u_{-1}\right) \\
& +I_{N}^{-\mu,-\mu}\left(\frac{1}{\Gamma(\gamma)} \int_{-1}^{x}(x-\theta)^{-\mu} e^{\gamma}(\theta) d \theta\right) \\
& +I_{N}^{-\mu,-\mu}\left(u_{-1}\right),
\end{aligned}
$$

where

$$
J_{1}(x)=I_{N}^{-\mu,-\mu} I_{1}(x), \quad J_{2}(x)=I_{N}^{-\mu,-\mu} I_{2}(x) .
$$

From (61)-(62), we have

$$
\begin{aligned}
e^{\gamma}(x)= & e(x)+\int_{-1}^{x} K_{1}(x, \theta) e(\theta) d \theta \\
& +\int_{-1}^{q x+q-1} K_{2}(x, \eta) e(\eta) d \eta \\
& +J_{1}(x)+J_{2}(x)+J_{3}(x)+J_{4}(x) \\
& +J_{5}(x)+J_{6}(x)+J_{7}(x), \\
e(x)= & \frac{1}{\Gamma(\gamma)} \int_{-1}^{x}(x-\theta)^{-\mu} e^{\gamma}(\theta) d \theta \\
& +J_{4}(x)+J_{8}(x),
\end{aligned}
$$

where

$$
\begin{aligned}
J_{3}(x)= & I_{N}^{-\mu,-\mu} D^{\gamma} u(x)-D^{\gamma} u(x), \\
J_{4}(x)= & I_{N}^{-\mu,-\mu} u(x)-u(x), \\
J_{5}(x)= & I_{N}^{-\mu,-\mu} u(q x+q-1)-u(q x+q-1), \\
J_{6}(x)= & I_{N}^{-\mu,-\mu} \int_{-1}^{x} K_{1}(x, \theta) e(\theta) d \theta \\
& \quad-\int_{-1}^{x} K(x, s) e(s) d s,
\end{aligned}
$$




$$
\begin{aligned}
J_{7}(x)= & I_{N}^{-\mu,-\mu} \int_{-1}^{q x+q-1} K_{2}(x, \eta) e(\eta) d \eta \\
& -\int_{-1}^{q x+q-1} K_{2}(x, \eta) e(\eta) d \eta, \\
J_{8}(x)= & I_{N}^{-\mu,-\mu}\left(\frac{1}{\Gamma(\gamma)} \int_{-1}^{x}(x-\theta)^{-\mu} e^{\gamma}(\theta) d \theta\right) \\
& -\frac{1}{\Gamma(\gamma)} \int_{-1}^{x}(x-\theta)^{-\mu} e^{\gamma}(\theta) d \theta .
\end{aligned}
$$

Due to (64), we obtain

$$
\begin{aligned}
e^{\gamma}(x)= & \frac{1}{\Gamma(\gamma)} \int_{-1}^{x}(x-\theta)^{-\mu} e^{\gamma}(\theta) d \theta \\
& +\frac{1}{\Gamma(\gamma)} \int_{-1}^{x} K_{1}(x, \theta) \int_{-1}^{\theta}(\theta-\tau)^{-\mu} e^{\gamma}(\tau) d \tau d \theta \\
& +\frac{1}{\Gamma(\gamma)} \int_{-1}^{q x+q-1} K_{2}(x, \eta) \int_{-1}^{\eta}(\eta-z)^{-\mu} \\
& \times e^{\gamma}(z) d z d \eta+H(x) \\
& +J_{1}(x)+J_{2}(x)+J_{3}(x)+J_{4}(x) \\
& +J_{5}(x)+J_{6}(x)+J_{7}(x),
\end{aligned}
$$

where

$$
\begin{aligned}
H(x)= & \int_{-1}^{x} K_{1}(x, \theta) \int_{-1}^{\theta}\left(J_{4}(\theta)+J_{8}(\theta)\right) d \theta \\
& +\frac{1}{\Gamma(\gamma)} \int_{-1}^{q x+q-1} K_{2}(x, \eta) \int_{-1}^{\eta}\left(J_{4}(\eta)+J_{8}(\eta)\right) d \eta \\
& +J_{4}(x)+J_{8}(x)
\end{aligned}
$$

Using the Dirichlet's formula which sates that

$$
\int_{-1}^{x} \int_{-1}^{\theta} \Phi(\theta, \tau) d \tau d \theta=\int_{-1}^{x} \int_{\tau}^{x} \Phi(\theta, \tau) d \theta d \tau
$$

provided that the integral exists, we obtain

$$
\begin{aligned}
& \frac{1}{\Gamma(\gamma)} \int_{-1}^{x} K_{1}(x, \theta) \int_{-1}^{\theta}(\theta-\tau)^{-\mu} e^{\gamma}(\tau) d \tau d \theta \\
& \quad=\frac{1}{\Gamma(\gamma)} \int_{-1}^{x}\left(\int_{\theta}^{x} K_{1}(x, \theta)(\theta-\tau)^{-\mu} d \theta\right) e^{\gamma}(\tau) d \tau \\
& \quad \leq \frac{1}{\Gamma(\gamma)} 2^{1-\mu} \max _{x, \tau \in[-1,1]}\left|\widetilde{K}_{1}(x, \tau)\right| \int_{-1}^{x}\left|e^{\gamma}(\tau)\right| d \tau \\
& \triangleq M_{1} \int_{-1}^{x}\left|e^{\gamma}(\tau)\right| d \tau,
\end{aligned}
$$

letting $\eta=q \tau+q-1, z=q \theta+q-1$, we have

$$
\begin{aligned}
& \frac{1}{\Gamma(\gamma)} \int_{-1}^{q x+q-1} K_{2}(x, \eta) \int_{-1}^{\eta}(\eta-z)^{-\mu} e^{\gamma}(z) d z d \eta \\
& =\frac{1}{\Gamma(\gamma)} q \int_{-1}^{x} K_{2}(x, q \tau+q-1) \\
& \times\left(\int_{-1}^{q \tau+q-1}(x-z)^{-\mu} e^{\gamma}(z) d z\right) d \tau \\
& =\frac{1}{\Gamma(\gamma)} q^{1+\gamma} \int_{-1}^{x} K_{2}(x, q \tau+q-1) \\
& \times\left(\int_{-1}^{\tau}(1-\theta)^{-\mu} e^{\gamma}(q \theta+q-1) d \theta\right) d \tau \\
& =\frac{1}{\Gamma(\gamma)} q^{1+\gamma} \int_{-1}^{x}\left(\int_{\theta}^{x} K_{2}(x, q \tau+q-1)\right. \\
& \left.\times(1-\theta)^{-\mu} d \tau\right) e^{\gamma} \\
& \times(q \theta+q-1) d \theta \\
& \leq \frac{1}{\Gamma(\gamma)} 2^{1-\mu} q^{1+\gamma} \max _{x, \tau \in[-1,1]}\left|K_{2}(x, \tau)\right| \\
& \times \int_{-1}^{x}\left|e^{\gamma}(q \theta+q-1)\right| d \theta \\
& \triangleq M_{2} \int_{-1}^{x}\left|e^{\gamma}(q \theta+q-1)\right| d \theta .
\end{aligned}
$$

Then, (67) gives

$$
\begin{aligned}
e^{\gamma}(x) \leq & \frac{1}{\Gamma(\gamma)} \int_{-1}^{x}(x-\theta)^{-\mu} e^{\gamma}(\theta) d \theta \\
& +M_{1} \int_{-1}^{x}\left|e^{\gamma}(\tau)\right| d \tau \\
& +M_{2} \int_{-1}^{x}\left|e^{\gamma}(q \theta+q-1)\right| d \theta \\
& +H(x)+J_{1}(x)+J_{2}(x)+J_{3}(x) \\
& +J_{4}(x)+J_{5}(x)+J_{6}(x)+J_{7}(x) .
\end{aligned}
$$

It follows from the Gronwall inequality that

$$
\left\|e^{\gamma}(x)\right\|_{L^{\infty}(I)} \leq \sum_{i=1}^{8}\left\|J_{i}(x)\right\|_{L^{\infty}(I)} .
$$

From (65), we have

$$
\|e(x)\|_{L^{\infty}(I)} \leq \sum_{i=1}^{8}\left\|J_{i}(x)\right\|_{L^{\infty}(I)} .
$$


We now apply Lemma 7 to obtain that

$\left\|J_{1}\right\|_{L^{\infty}(I)}$

$$
\begin{aligned}
& \leq \begin{cases}C N^{(1 / 2)-\mu} \max _{0 \leq i \leq N}\left|I_{i, 1}\right|, & 0<\mu<\frac{1}{2}, \\
C \log N \max _{0 \leq i \leq N}\left|I_{i, 1}\right|, & \frac{1}{2} \leq \mu<1,\end{cases} \\
& \leq \begin{cases}C N^{(1 / 2)-\mu-m} K_{1}^{*}\left(\|u\|_{L^{2}(I)}+\left\|e^{\gamma}(x)\right\|_{L^{\infty}(I)}\right), & 0<\mu<\frac{1}{2}, \\
C N^{-m} \log N K_{1}^{*}\left(\|u\|_{L^{2}(I)}+\left\|e^{\gamma}(x)\right\|_{L^{\infty}(I)}\right), & \frac{1}{2} \leq \mu<1,\end{cases}
\end{aligned}
$$

$\left\|J_{2}\right\|_{L^{\infty}(I)}$

$$
\begin{aligned}
& \leq \begin{cases}C N^{(1 / 2)-\mu} \max _{0 \leq i \leq N}\left|I_{i, 2}\right|, & 0<\mu<\frac{1}{2}, \\
C \log N \max _{0 \leq i \leq N}\left|I_{i, 1}\right|, & \frac{1}{2} \leq \mu<1,\end{cases} \\
& \leq \begin{cases}C N^{(1 / 2)-\mu-m} K_{2}^{*}\left(\|u\|_{L^{2}(I)}+\left\|e^{\gamma}(x)\right\|_{L^{\infty}(I)}\right), & 0<\mu<\frac{1}{2}, \\
C N^{-m} \log N K_{2}^{*}\left(\|u\|_{L^{2}(I)}+\left\|e^{\gamma}(x)\right\|_{L^{\infty}(I)}\right), & \frac{1}{2} \leq \mu<1 .\end{cases}
\end{aligned}
$$

Due to Lemma 2,

$$
\begin{aligned}
& \left\|J_{3}\right\|_{L^{\infty}(I)} \leq \begin{cases}C N^{1-\mu-m}\left|D^{\gamma} u\right|_{H_{\omega^{c}}^{m, N}(I)}, & 0<\mu<\frac{1}{2}, \\
C N^{(1 / 2)-m} \log N\left|D^{\gamma} u\right|_{H_{\omega^{c}}^{m^{\prime} N}(I)}, & \frac{1}{2} \leq \mu<1,\end{cases} \\
& \left\|J_{4}\right\|_{L^{\infty}(I)} \leq \begin{cases}C N^{1-\mu-m}|u|_{H_{\omega^{c}}^{m^{c} N}(I)}, & 0<\mu<\frac{1}{2}, \\
C N^{(1 / 2)-m} \log N|u|_{H_{\omega^{c}}^{m_{N}}(I)}, & \frac{1}{2} \leq \mu<1,\end{cases} \\
& \left\|J_{5}\right\|_{L^{\infty}(I)} \leq \begin{cases}C N^{1-\mu-m}|u|_{H_{\omega^{c}}^{m_{c} N}(I)}, & 0<\mu<\frac{1}{2}, \\
C N^{(1 / 2)-m} \log N|u|_{H_{\omega^{c}}^{m_{N} N}(I)}, & \frac{1}{2} \leq \mu<1 .\end{cases}
\end{aligned}
$$

By virtue of Lemma 2 (33b) with $m=1$,

$$
\begin{array}{r}
\left\|J_{6}\right\|_{L^{\infty}(I)} \leq \begin{cases}C N^{-\mu}\|e\|_{L^{\infty}(I)}, & 0<\mu<\frac{1}{2}, \\
C N^{-(1 / 2)}\|e\|_{L^{\infty}(I)}, & \frac{1}{2} \leq \mu<1,\end{cases} \\
\left\|J_{7}\right\|_{L^{\infty}(I)} \leq \begin{cases}C N^{-\mu}\|e\|_{L^{\infty}(I)}, & 0<\mu<\frac{1}{2}, \\
C N^{-(1 / 2)}\|e\|_{L^{\infty}(I)}, & \frac{1}{2} \leq \mu<1 .\end{cases}
\end{array}
$$

We now estimate the term $J_{8}(x)$. It follows from Lemmas 5 and 6 with $K(x, \tau)=1 / \Gamma(\gamma)$ that

$$
\begin{aligned}
\left\|J_{5}\right\|_{L^{\infty}(I)} & =\left\|\left(I_{N}^{-\mu,-\mu}-I\right) \mathscr{M} e^{\gamma}\right\|_{L^{\infty}(I)} \\
& =\left\|\left(I_{N}^{-\mu,-\mu}-I\right)\left(\mathscr{M} e^{\gamma}-\mathscr{T}_{N} M e^{\gamma}\right)\right\|_{L^{\infty}(I)} \\
& \leq\left(1+\left\|I_{N}^{-\mu,-\mu}\right\|_{L^{\infty}(I)}\right) C N^{-k}\left\|M e^{\gamma}\right\|_{0, \kappa} \\
& \leq \begin{cases}C N^{(1 / 2)-\mu-\kappa}\left\|e^{\gamma}\right\|_{L^{\infty}(I)}, & 0<\mu<\frac{1}{2}, \\
C N^{-\kappa} \log N\left\|e^{\gamma}\right\|_{L^{\infty}(I)}, & \frac{1}{2} \leq \mu<1,\end{cases}
\end{aligned}
$$

where in the last step we have used Lemma 6 under the following assumption:

$$
\begin{gathered}
\frac{1}{2}-\mu<\kappa<1-\mu, \quad \text { when } 0<\mu<\frac{1}{2}, \\
0<\kappa<1-\mu, \quad \text { when } \frac{1}{2} \leq \mu<1 .
\end{gathered}
$$

Provided that $N$ is sufficiently large, combining (75), (76), (77), and (78) gives

$$
\begin{aligned}
& \left\|U^{\gamma}(x)-u^{\gamma}(x)\right\|_{L^{\infty}(I)} \\
& \leq \begin{cases}C N^{(1 / 2)-\mu-m}\left(K^{*}\|u\|_{L^{2}(I)}+N^{(1 / 2)} \mathcal{U}\right), & 0<\mu<\frac{1}{2}, \\
C N^{-m} \log N\left(K^{*}\|u\|_{L^{2}(I)}+N^{(1 / 2)} u\right), & \frac{1}{2} \leq \mu<1,\end{cases} \\
& \quad\|U(x)-u(x)\|_{L^{\infty}(I)} \\
& \quad \begin{cases}C N^{(1 / 2)-\mu-m}\left(K^{*}\|u\|_{L^{2}(I)}+N^{(1 / 2)} \mathcal{U}\right), & 0<\mu<\frac{1}{2}, \\
C N^{-m} \log N\left(K^{*}\|u\|_{L^{2}(I)}+N^{(1 / 2)} U\right), & \frac{1}{2} \leq \mu<1 .\end{cases}
\end{aligned}
$$

Using $\gamma=1-\mu$, we have the desired estimates (46) and (47).

Next, we will give the error estimates in $L_{\omega^{-\mu,-\mu}}^{2}$ space.

Theorem 10. If the hypotheses given in Theorem 9 hold, then $\left\|U^{\gamma}(x)-u^{\gamma}(x)\right\|_{L_{\omega^{2}}^{2}-\mu, \mu}(I)$

$$
\leq \begin{cases}C N^{-m}\left(V_{1}+N^{\gamma-(1 / 2)-\kappa} V_{2}+N^{\gamma-\kappa} \mathcal{U}\right), & \frac{1}{2}<\gamma<1, \\ C N^{-m}\left(V_{1}+N^{-\kappa} \log N V_{2}+N^{(1 / 2)-\kappa} \log N U\right), & 0<\gamma \leq \frac{1}{2},\end{cases}
$$

$\|U(x)-u(x)\|_{L_{\omega}^{2}-\mu,-\mu}(I)$

$$
\leq \begin{cases}C N^{-m}\left(V_{1}+N^{\gamma-(1 / 2)-\kappa} V_{2}+N^{\gamma-\kappa} U\right), & \frac{1}{2}<\gamma<1, \\ C N^{-m}\left(V_{1}+N^{-\kappa} \log N V_{2}+N^{(1 / 2)-\kappa} \log N \mathcal{U}\right), & 0<\gamma \leq \frac{1}{2},\end{cases}
$$


for any $\kappa \in(0, \gamma)$ provided that $N$ is sufficiently large and $C$ is a constant independent of $N$, where

$$
\begin{gathered}
V_{1}=K^{*}\left(\|u\|_{L^{2}(I)}+\left|D^{\gamma} u\right|_{H_{\omega^{c}}^{m ; N}(I)}+|u|_{H_{\omega^{c}}^{m ; N}(I)}\right), \\
V_{2}=K^{*}\|u\|_{L^{2}(I)} .
\end{gathered}
$$

Proof. By using the generalization of Gronwalls Lemma 4 and the Hardy inequality Lemma 8, it follows from (72) that

$$
\begin{gathered}
\left\|e^{\gamma}\right\|_{L_{\omega^{-}-\mu, \mu}^{2}(I)} \leq C \sum_{i=1}^{8}\left\|J_{i}\right\|_{L_{\omega^{2}-\mu,-\mu}^{2}(I)}, \\
\|e\|_{L_{\omega^{2}-\mu,-\mu}^{2}(I)} \leq C \sum_{i=1}^{8}\left\|J_{i}\right\|_{L_{\omega^{-\mu},-\mu}^{2}(I)} .
\end{gathered}
$$

Now, using Lemma 7, we have

$$
\begin{aligned}
\left\|J_{1}\right\|_{L_{\omega^{-\mu},-\mu}^{2}(I)} & \leq C \max _{x \in[-1,1]}|I(x)| \\
& \leq C N^{-m} K_{1}^{*}\left(\|u\|_{L^{2}(I)}+\|e\|_{L^{\infty}(I)}\right), \\
\left\|J_{2}\right\|_{L_{\omega^{2}-\mu, \mu}^{2}(I)} & \leq C \max _{x \in[-1,1]}|I(x)| \\
& \leq C N^{-m} K_{2}^{*}\left(\|u\|_{L^{2}(I)}+\|e\|_{L^{\infty}(I)}\right) .
\end{aligned}
$$

By the convergence result in Theorem $9(m=1)$, we have

$$
\|e\|_{L^{\infty}(I)} \leq C\left(\left|D^{\gamma} u\right|_{H_{\omega^{c}}^{m ; N}(I)}+|u|_{H_{\omega^{c}}^{m ; N}(I)}+\|u\|_{L^{2}(I)}\right) .
$$

So that

$$
\begin{aligned}
& \left\|J_{1}\right\|_{L_{\omega^{-}-\mu_{-}-\mu}(I)} \\
& \leq C N^{-m} K_{1}^{*}\left(\left|D^{\gamma} u\right|_{H_{\omega^{c}}^{m ; N}(I)}+|u|_{H_{\omega^{c}}^{m ; N}(I)}+\|u\|_{L^{2}(I)}\right), \\
& \left\|J_{2}\right\|_{L_{\omega}^{2}-\mu,-\mu}(I) \\
& \leq C N^{-m} K_{2}^{*}\left(\left|D^{\gamma} u\right|_{H_{\omega^{c}}^{m ; N}(I)}+|u|_{H_{\omega^{c}}^{m ; N}(I)}+\|u\|_{L^{2}(I)}\right) .
\end{aligned}
$$

Due to Lemma 2 (33a),

$$
\begin{gathered}
\left\|J_{3}\right\|_{L_{\omega^{2}-\mu}^{2}-\mu} \leq C N^{-m}\left|D^{\gamma} u\right|_{H_{\omega^{2}}^{m_{i} N}(I)}, \\
\left\|J_{4}\right\|_{L_{\omega^{-\mu}-\mu}^{2}(I)} \leq C N^{-m}|u|_{H_{\omega^{2}}^{m_{i} N}(I)}, \\
\left\|J_{5}\right\|_{L_{\omega^{-\mu}-\mu}^{2}-\mu} \leq C N^{-m}|u|_{H_{\omega^{2}}^{m i N}(I)} .
\end{gathered}
$$

By virtue of Lemma 2 (33a) with $m=1$,

$$
\begin{aligned}
& \left\|J_{6}\right\|_{L_{\omega^{-\mu},-\mu}^{2}(I)} \leq C N^{-1}\left|\int_{-1}^{x} K_{1}(x, \theta) e(\theta) d \theta\right|_{\substack{H_{\omega^{1}-\mu,-\mu}^{1 ; N}(I) \\
\text {. }}} \\
& \leq C N^{-1}\|e\|_{L_{\omega^{-}-\mu,-\mu}^{2}(I)}, \\
& \left\|J_{7}\right\|_{L_{\omega^{-}-\mu,-\mu}(I)} \leq C N^{-1}\left|\int_{-1}^{q x+q-1} K_{2}(x, \eta) e(\eta) d \eta\right|_{\substack{H_{\omega^{1},-\mu,-\mu},(I) \\
\mid}} \\
& \leq C N^{-1}\|e\|_{L_{\omega^{-\mu},-\mu}^{2}(I)} \text {. }
\end{aligned}
$$

Finally, it follows from Lemmas 5 and 7 that

$$
\begin{aligned}
& \left\|J_{8}\right\|_{L_{\omega^{-} \mu,-\mu}^{2}(I)}=\left\|\left(I_{N}^{-\mu,-\mu}-I\right) M e^{\gamma}\right\|_{L_{\omega^{-\mu},-\mu}^{2}(I)} \\
& =\left\|\left(I_{N}^{-\mu,-\mu}-I\right)\left(\mathscr{M} e^{\gamma}-\mathscr{T}_{N} e^{\gamma}\right)\right\|_{L_{\omega^{-}-\mu,-\mu}^{2}(I)} \\
& \leq\left\|I_{N}^{-\mu,-\mu}\left(M e^{\gamma}-\mathscr{T}_{N} e^{\gamma}\right)\right\|_{L_{\omega^{-}-\mu,-\mu}^{2}(I)} \\
& +\left\|\mathscr{M} e^{\gamma}-\mathscr{T}_{N} e^{\gamma}\right\|_{L_{\omega}^{2}-\mu,-\mu}(I) \\
& \leq C\left\|\mathscr{M} e^{\gamma}-\mathscr{T}_{N} e^{\gamma}\right\|_{L^{\infty}(I)} \\
& \leq C N^{-\kappa}\left\|\mathscr{M} e^{\gamma}\right\|_{0, \kappa} \\
& \leq C N^{-\kappa}\left\|e^{\gamma}\right\|_{L^{\infty}(I)},
\end{aligned}
$$

where in the last step we used Lemma 6 for any $\kappa \in(0,1-\mu)$. By the convergence result in Theorem 9, we obtain that

$$
\begin{aligned}
& \left\|J_{8}\right\|_{L_{\omega^{-}-\mu, \mu}^{2}(I)} \\
& \quad \leq \begin{cases}C N^{1 / 2-\mu-m-\kappa}\left(K^{*}\|u\|_{L^{2}(I)}+N^{1 / 2} U\right), & 0<\mu<\frac{1}{2}, \\
C N^{-m-\kappa} \log N\left(K^{*}\|u\|_{L^{2}(I)}+N^{1 / 2} \mathcal{U}\right), & \frac{1}{2} \leq \mu<1,\end{cases}
\end{aligned}
$$

for $N$ sufficiently large and for any $\kappa \in(0,1-\mu)$. The desired estimates (81) follows from the previous estimates and (83) with $\gamma=1-\mu$.

\section{Algorithm Implementation and Numerical Experiments}

In this subsection, we present the numerical results obtained by the proposed collocation spectral method. The estimates in Section 4 indicates that the convergence of numerical solutions is exponential if the exact solution is smooth. To confirm the theoretical prediction, a numerical experiment is carried out by considering the following example.

Example 1. Consider the following fractional integrodifferential equation:

$$
\begin{aligned}
& D^{0.75} y(t)= \frac{6 t^{2.25}}{\Gamma(3.25)}-\frac{t^{2} e^{t}}{5} y(t)-\frac{(q t)^{2}}{5} y(q t) \\
&+\int_{0}^{t} e^{t} s y(s) d s+\int_{0}^{q t} \tau y(\tau) d \tau, \quad t \in[0,1], \\
& y(0)=0 .
\end{aligned}
$$

The corresponding exact solution is given by $y(t)=t^{3}$.

Figure 1 presents the approximate and exact solutions on the left-hand side and presents the approximate and 


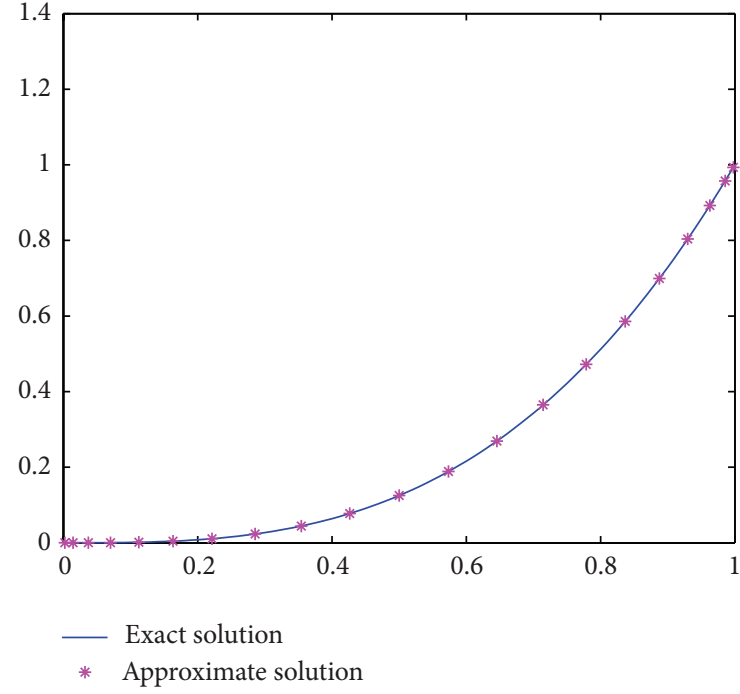

(a)

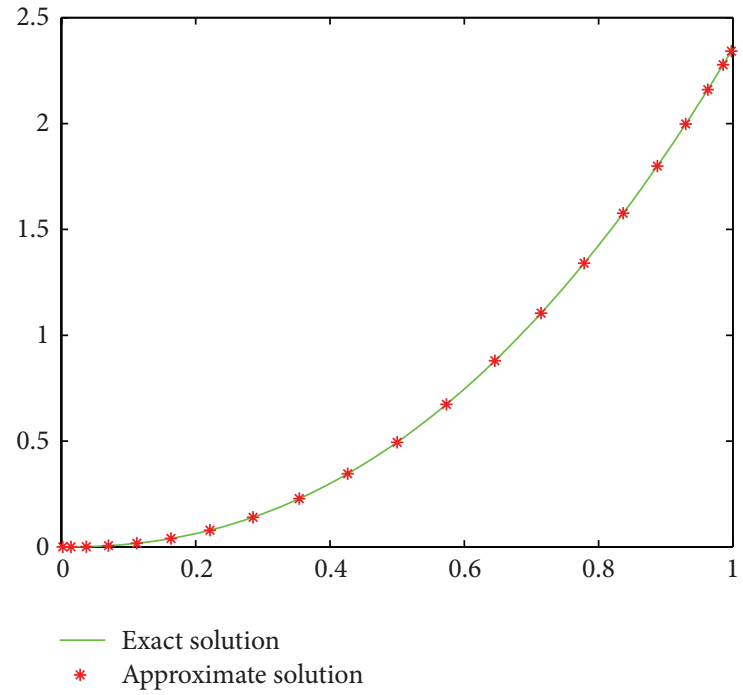

(b)

FIGURE 1: Example 1: comparison between approximate solution and exact solution $y(t)$ (a) and approximate fraction derivative and exact derivative $D^{0.75} y(t)(\mathrm{b})$.

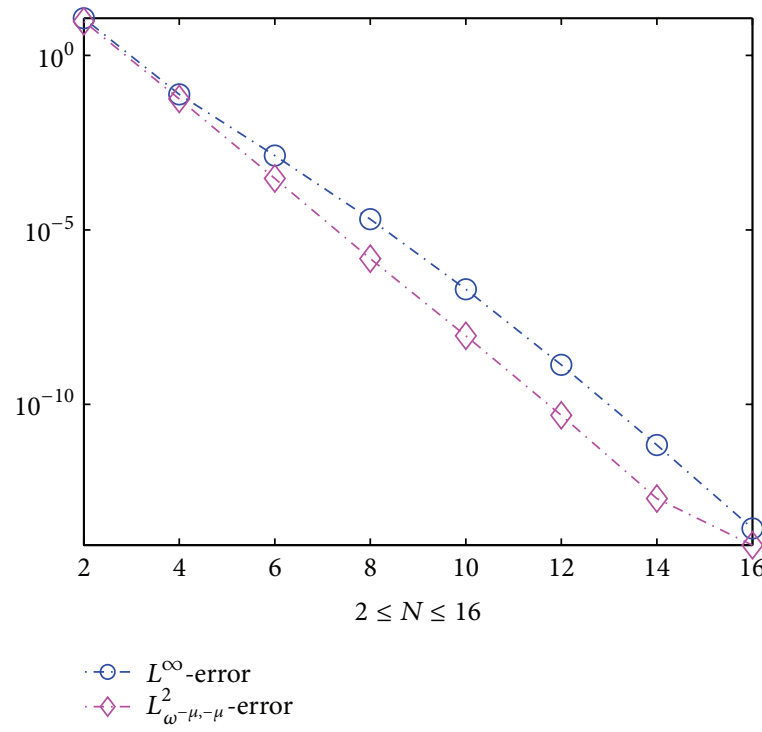

(a)

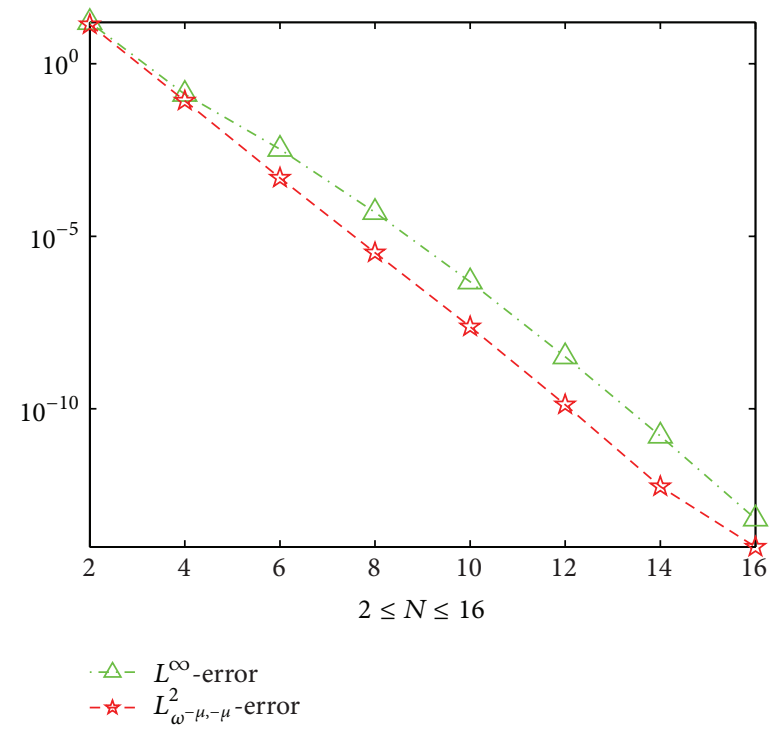

(b)

FIGURE 2: Example 1: the errors of numerical and exact solution $y(t)$ (a) and the errors of numerical and exact solution $D^{0.75} y(t)(\mathrm{b})$ versus the number of collocation points in $L^{\infty}$ and $L_{\omega}^{2}$ norms.

exact derivatives on the right-hand side, which are found in excellent agreement. In Figure 2, the numerical errors are plotted for $2 \leq N \leq 20$ in both $L^{\infty}$ and $L_{\omega^{-\mu,-\mu}}^{2}$ norms. As expected, an exponential rate of convergence is observed for the problem, which confirmed our theoretical predictions.

Example 2. Consider the following fractional integrodifferential equation:

$$
\begin{gathered}
D^{\alpha} y(t)=2 t-y(t)+y(q t)+t(1+2 t) \int_{0}^{t} e^{s(t-s)} y(s) d s \\
+\int_{0}^{q t} q t e^{\tau(q t-\tau)} y(\tau) d \tau, \quad t \in[0,1], \\
y(0)=1,
\end{gathered}
$$

when $\alpha=1$, the exact solution of $(92)$ is $y(t)=e^{t^{2}}$. 


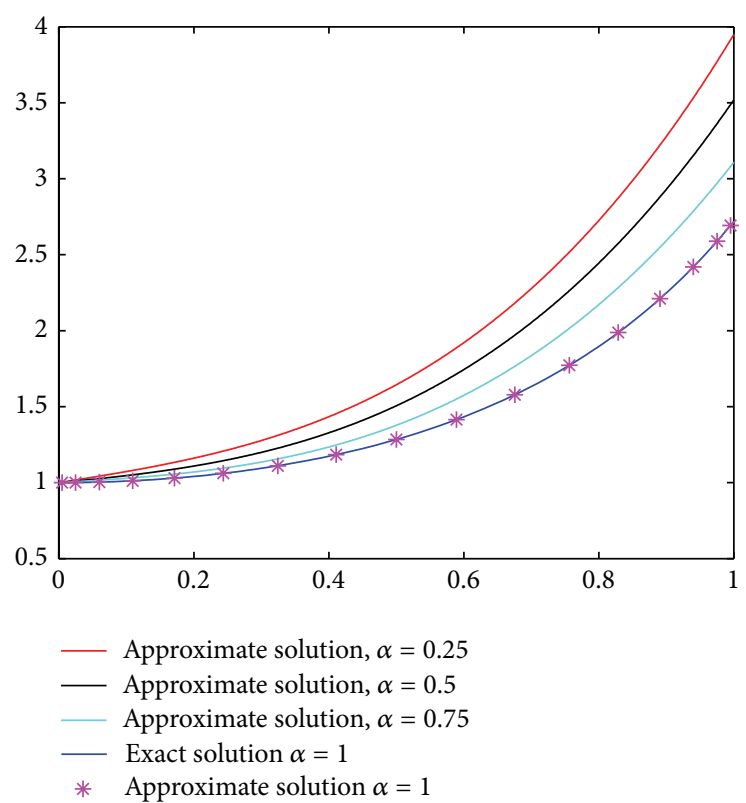

(a)

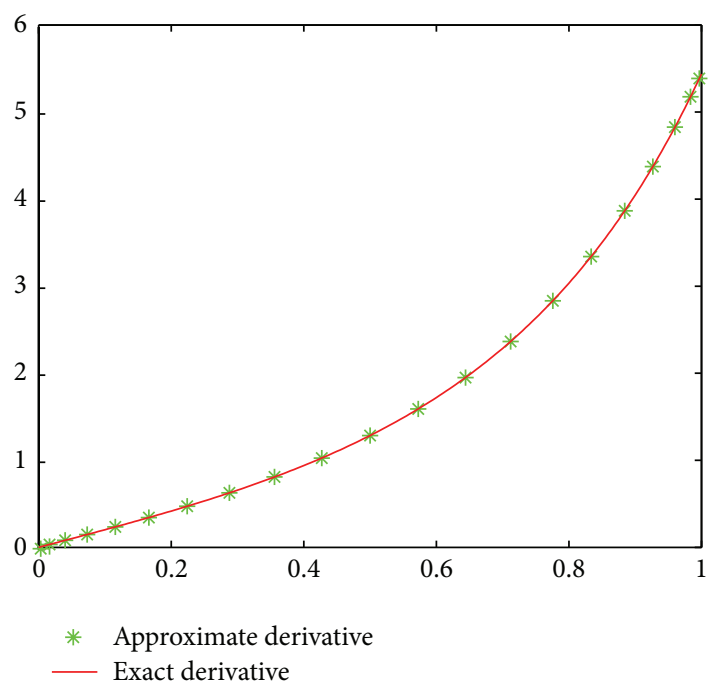

(b)

FIGURE 3: Example 2: approximation solutions with different $\alpha$ and exact solution of $y(t)$ with $\alpha=1$ (a). Comparison between approximate solution and exact solution of $y^{\prime}(t)$.

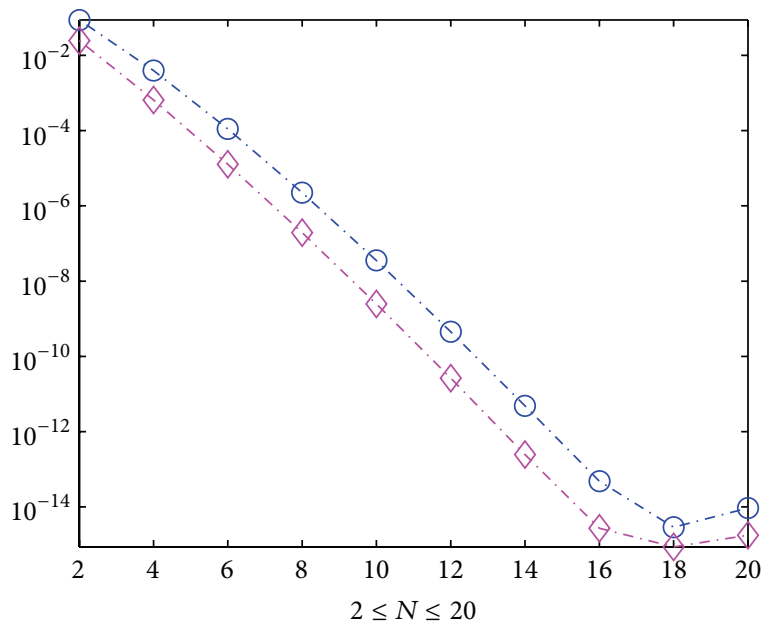

$\bigcirc-L^{\infty}$

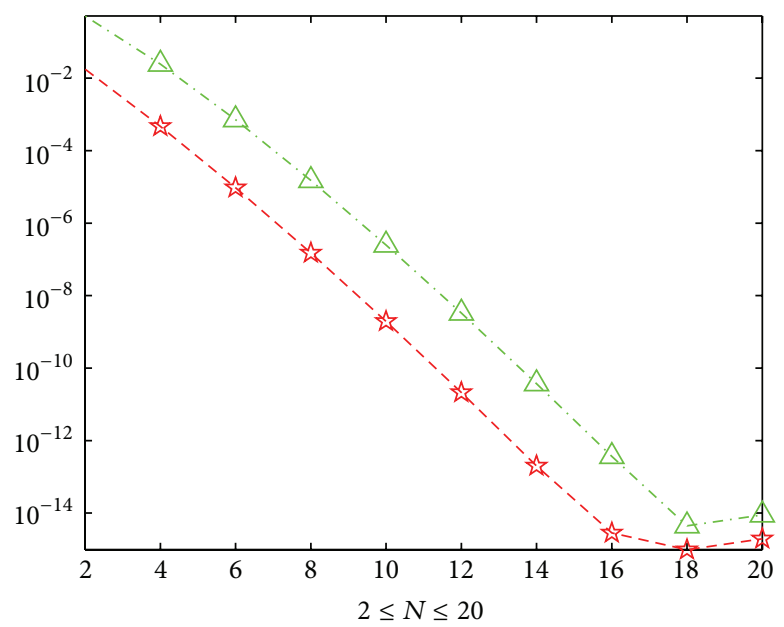

$\triangle-L^{\infty}$$$
-\frac{1}{h}-L_{\omega}^{2}
$$

(a)

(b)

FiguRE 4: Example 2: the errors of numerical and exact solution $y(t)$ (a) and the errors of numerical and exact solution $y^{\prime}(t)$ (b) versus the number of collocation points in $L^{\infty}$ and $L_{\omega}^{2}$ norms.

In the only case of $\alpha=1$, we know the exact solution. We have reported the obtained numerical results for $N=20$ and $\alpha=0.25,0.5,0.75$, and 1 in Figure 3. We can see that, as $\alpha$ approaches 1, the numerical solutions converge to the analytical solution $y(t)=e^{t^{2}}$; that is, in the limit, the solution of fractional integrodifferential equations approach to that of the integer order integrodifferential equations. In Figure 4, we plot the resulting errors versus the number $N$ of the steps. This figure shows the exponential rate of convergence predicted by the proposed method.

\section{Conclusions and Future Work}

This paper proposes a spectral Jacobi-collocation approximation for fractional order integrodifferential equations of Volterra type with pantograph delay. The most important 
contribution of this work is that we are able to demonstrate rigorously that the errors of spectral approximations decay exponentially in both infinity and weighted norms, which is a desired feature for a spectral method.

We only investigated the case of pantograph delay in the present work, with the availability of this methodology, it will be possible to generalize this algorithm to solve the same problem in semi-infinite interval based on generalized Laguerre [35] and modified generalized Laguerre polynomials.

\section{Acknowledgments}

The authors are grateful to the referees for many useful suggestions. The work was supported by NSFC Project (11301446, 11031006), China Postdoctoral Science Foundation Grant (2013M531789), Project of Scientific Research Fund of Hunan Provincial Science and Technology Department (2013RS4057), and the Research Foundation of Hunan Provincial Education Department (13B116).

\section{References}

[1] J. H. He, "Nonlinear oscillation with fractional derivative and its Cahpinpalications," in Proceedings of the International Conference on Vibrating Engineering, pp. 288-291, Dalian, China, 1998.

[2] J. H. He, "Some applications of nonlinear fractional differential equations and therir approximations," Bulletin of Science, Technology and Society, vol. 15, pp. 86-90, 1999.

[3] I. Podlubny, Fractional Differential Equations, Academic Press, NewYork, NY, USA, 1999.

[4] F. Mainardi, Fractional Calculus Continuum Mechanics, Springer, Berlin, Germany, 1997.

[5] W. M. Ahmad and R. El-Khazali, "Fractional-order dynamical models of love," Chaos, Solitons and Fractals, vol. 33, no. 4, pp. 1367-1375, 2007.

[6] F. Huang and F. Liu, "The time fractional diffusion equation and the advection-dispersion equation," The ANZIAM Journal, vol. 46, no. 3, pp. 317-330, 2005.

[7] Y. Luchko and R. Gorenflo, The Initial Value Problem for Some Fractional Differential Equations with the Caputo Derivatives, Preprint series A08-98, Fachbreich Mathematik und Informatik, Freic Universitat, Berlin, Germany, 1998.

[8] N. T. Shawagfeh, "Analytical approximate solutions for nonlinear fractional differential equations," Applied Mathematics and Computation, vol. 131, no. 2-3, pp. 517-529, 2002.

[9] S. G. Samko, A. A. Kilbas, and O. I. Marichev, Fractional Integrals and Derivatives: Theory and Applications, Gordon and Breach Science, Yverdon, Switzerland, 1993.

[10] O. P. Agrawal and P. Kumar, "Comparison of five numerical schemes for fractional differential equations," in Advances in Fractional Calculus: Theoretical Developments and Applications in Physics and Engineering, J. Sabatier et al., Ed., pp. 43-60, Springer, Berlin, Germany, 2007.

[11] M. M. Khader and A. S. Hendy, "The approximate and exact solutions of the fractional-order delay differential equations using Legendre seudospectral method," International Journal of Pure and Applied Mathematics, vol. 74, no. 3, pp. 287-297, 2012.
[12] A. Saadatmandi and M. Dehghan, "A Legendre collocation method for fractional integro-differential equations," Journal of Vibration and Control, vol. 17, no. 13, pp. 2050-2058, 2011.

[13] E. A. Rawashdeh, "Legendre wavelets method for fractional integro-differential equations," Applied Mathematical Sciences, vol. 5, no. 2, pp. 2467-2474, 2011.

[14] M. Rehman and R. A. Khan, "The Legendre wavelet method for solving fractional differential equations," Communications in Nonlinear Science and Numerical Simulation, vol. 16, no. 11, pp. 4163-4173, 2011.

[15] A. H. Bhrawy and M. A. Alghamdi, "A shifted Jacobi-GaussLobatto collocation method for solving nonlinear fractional Langevin equation involving two fractional orders in different intervals," Boundary Value Problems, vol. 1, no. 62, pp. 1-13, 2012.

[16] E. H. Doha, A. H. Bhrawy, and S. S. Ezz-Eldien, "Efficient Chebyshev spectral methods for solving multi-term fractional orders differential equations," Applied Mathematical Modelling, vol. 35, no. 12, pp. 5662-5672, 2011.

[17] E. H. Doha, A. H. Bhrawy, and S. S. Ezz-Eldien, "A Chebyshev spectral method based on operational matrix for initial and boundary value problems of fractional order," Computers \& Mathematics with Applications, vol. 62, no. 5, pp. 2364-2373, 2011.

[18] N. H. Sweilam and M. M. Khader, "A Chebyshev pseudospectral method for solving fractional-order integro-differential equations," The ANZIAM Journal, vol. 51, no. 4, pp. 464-475, 2010.

[19] A. H. Bhrawy, A. S. Alofi, and S. S. Ezz-Eldien, "A quadrature tau method for fractional differential equations with variable coefficients," Applied Mathematics Letters, vol. 24, no. 12, pp. 2146-2152, 2011.

[20] A. H. Bhrawy and M. Alshomrani, "A shifted Legendre spectral method for fractional-order multi-point boundary value problems," Advances in Difference Equations, vol. 2012, article 8, 2012.

[21] A. Saadatmandi and M. Dehghan, "A new operational matrix for solving fractional-order differential equations," Computers \& Mathematics with Applications, vol. 59, no. 3, pp. 1326-1336, 2010.

[22] A. H. Bhrawy, M. M. Tharwat, and A. Yildirim, "A new formula for fractional integrals of Chebyshev polynomials: application for solving multi-term fractional differential equations," Applied Mathematical Modelling, vol. 37, no. 6, pp. 4245-4252, 2013.

[23] A. H. Bhrawy and A. S. Alofi, "The operational matrix of fractional integration for shifted Chebyshev polynomials," Applied Mathematics Letters, vol. 26, no. 1, pp. 25-31, 2013.

[24] E. H. Doha, A. H. Bhrawy, and S. S. Ezz-Eldien, "A new Jacobi operational matrix: an application for solving fractional differential equations," Applied Mathematical Modelling, vol. 36, no. 10, pp. 4931-4943, 2012.

[25] Y. Chen and T. Tang, "Convergence analysis of the Jacobi spectral-collocation methods for Volterra integral equations with a weakly singular kernel," Mathematics of Computation, vol. 79, no. 269, pp. 147-167, 2010.

[26] Y. Wei and Y. Chen, "Convergence analysis of the spectral methods for weakly singular Volterra integro-differential equations with smooth solutions," Advances in Applied Mathematics and Mechanics, vol. 4, no. 1, pp. 1-20, 2012.

[27] C. Canuto, M. Y. Hussaini, A. Quarteroni, and T. A. Zang, Spectral Methods, Fundamentals in Single Domains, Springer, Berlin, Germany, 2006. 
[28] G. Mastroianni and D. Occorsio, "Optimal systems of nodes for Lagrange interpolation on bounded intervals. A survey," Journal of Computational and Applied Mathematics, vol. 134, no. 1-2, pp. 325-341, 2001.

[29] D. Henry, Geometric Theory of Semilinear Parabolic Equations, Springer, Berlin, Germany, 1989.

[30] D. L. Ragozin, "Polynomial approximation on compact manifolds and homogeneous spaces," Transactions of the American Mathematical Society, vol. 150, pp. 41-53, 1970.

[31] D. L. Ragozin, "Constructive polynomial approximation on spheres and projective spaces," Transactions of the American Mathematical Society, vol. 162, pp. 157-170, 1971.

[32] D. Colton and R. Kress, Inverse Coustic and Electromagnetic Scattering Theory, Applied Mathematical Sciences, Springer, Berlin, Germany, 2nd edition, 1998.

[33] P. Nevai, "Mean convergence of Lagrange interpolation. III," Transactions of the American Mathematical Society, vol. 282, no. 2, pp. 669-698, 1984.

[34] A. Kufner and L. E. Persson, Weighted Inequalities of Hardy Type, World Scientific, New York, NY, USA, 2003.

[35] D. Baleanu, A. H. Bhrawy, and T. M. Taha, "Two efficient generalized Laguerre spectral algorithms for fractional initial value problems," Abstract and Applied Analysis, vol. 2013, Article ID 546502, 10 pages, 2013. 


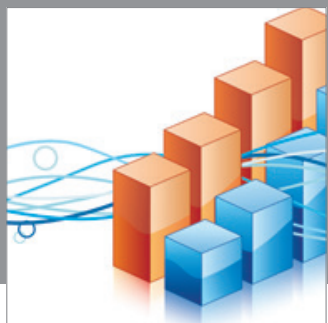

Advances in

Operations Research

mansans

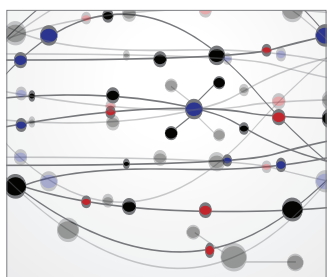

The Scientific World Journal
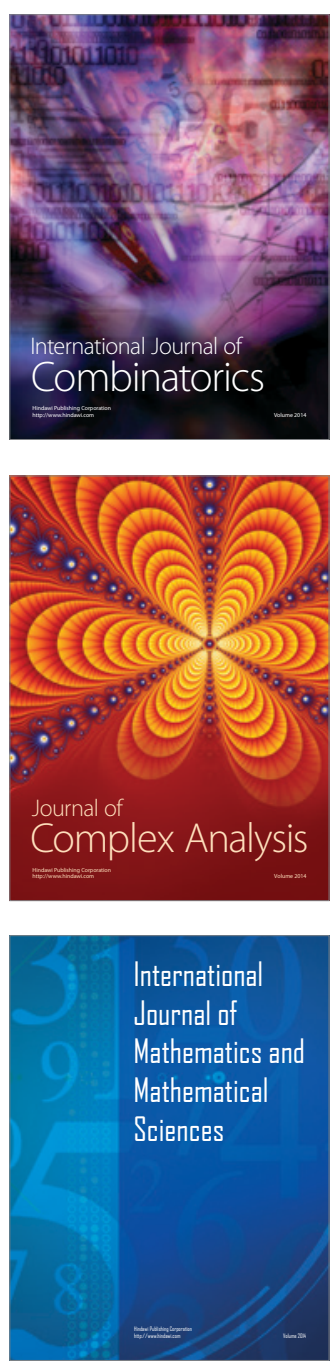
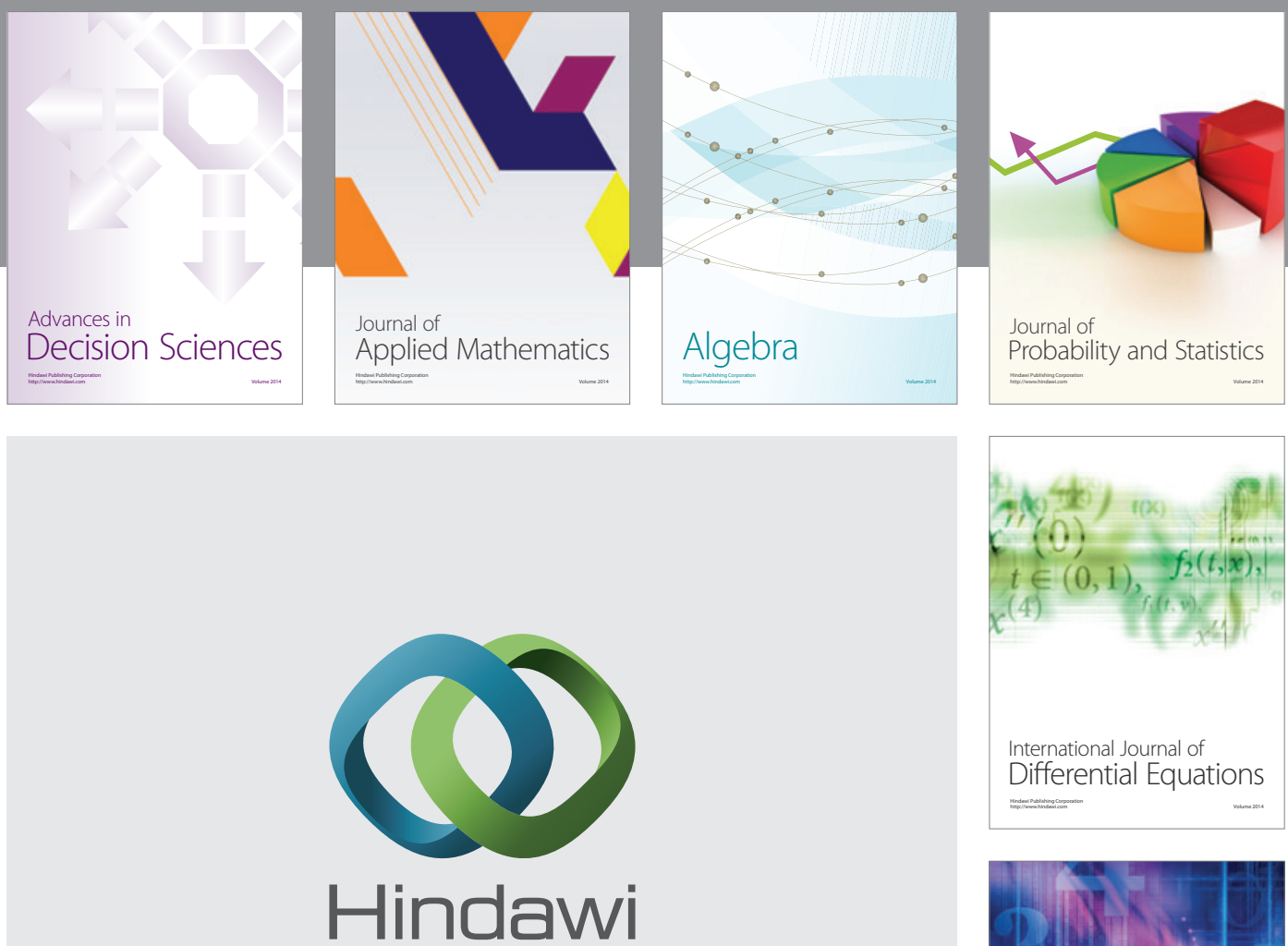

Submit your manuscripts at http://www.hindawi.com
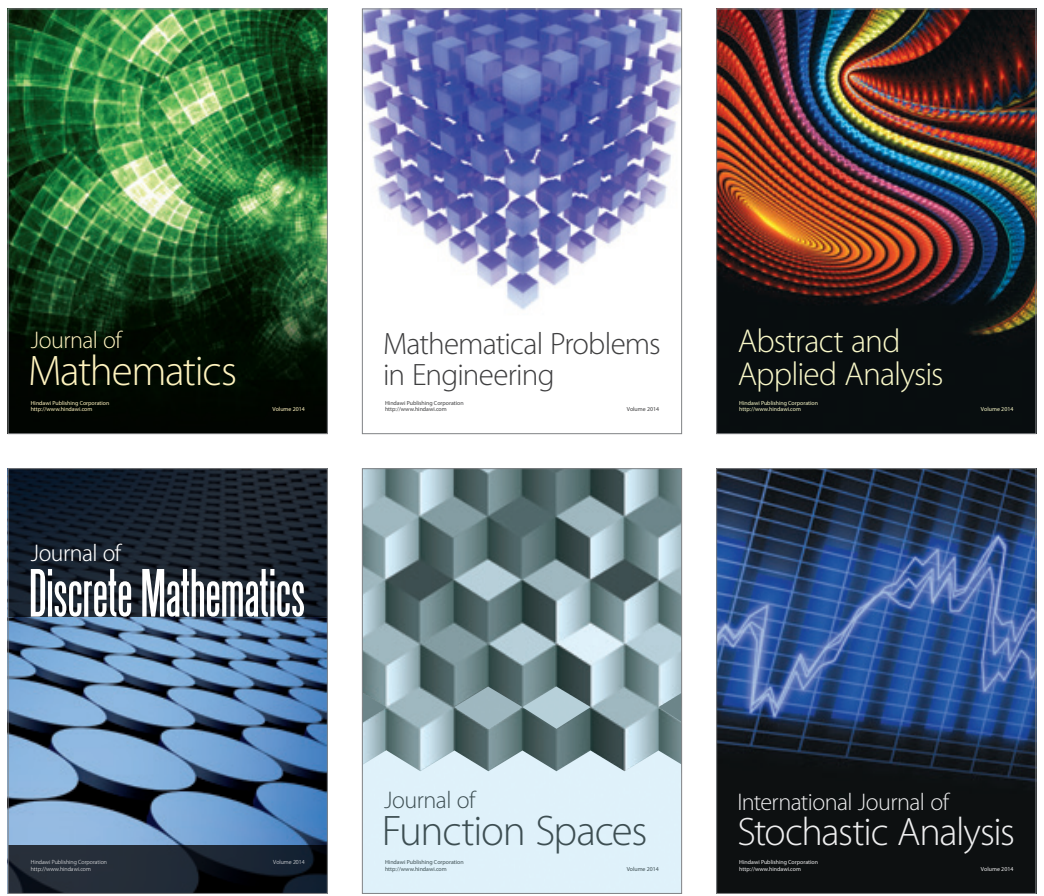

Journal of

Function Spaces

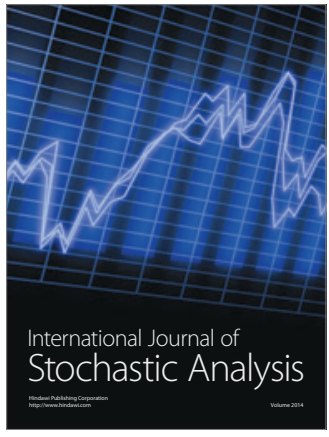

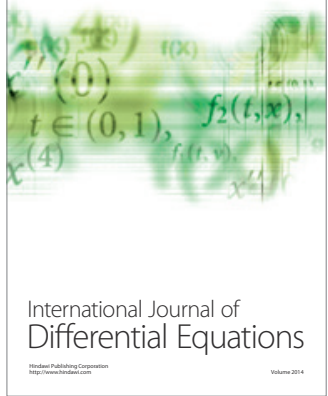
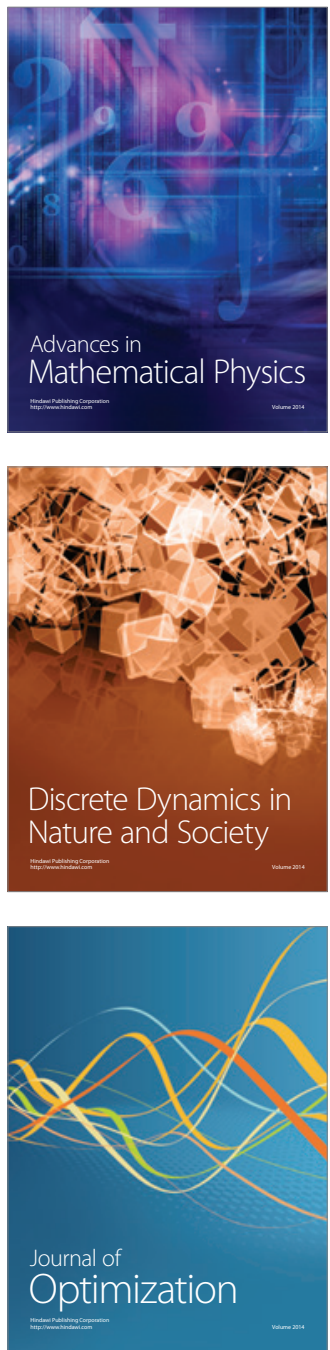